\title{
Efficacy and Safety of Ultrasound-guided Percutaneous Transthoracic Needle Biopsy for Peripheral Pulmonary Lesions: Comparison Between 16-gauge and 18-gauge Core Biopsy Needle- a Propensity Score Matching Analysis
}

\section{Weijun Huang}

First People's Hospital of Foshan

Jieyi Ye

First People's Hospital of Foshan

\section{Yide Qiu}

First People's Hospital of Foshan

\section{Weiwei Peng}

First People's Hospital of Foshan

Ninghui Lan

First People's Hospital of Foshan

Weizhen Cui

First People's Hospital of Foshan

Ting Huang

First People's Hospital of Foshan

\section{Yinghui Ou}

First People's Hospital of Foshan

Yingjia Li ( $\square$ lyjia@fimmu.com)

Department of Medical Ultrasonics, Nanfang Hospital, Southern Medical University, No. 1838, North Guangzhou Avenue, Guangzhou 510515, Guangdong, China.

\section{Research Article}

Keywords: efficacy, safety, ultrasound-guided biopsy, peripheral pulmonary lesion, propensity score matching analysis

Posted Date: November 25th, 2020

DOI: https://doi.org/10.21203/rs.3.rs-110059/v1 
License: (c) (i) This work is licensed under a Creative Commons Attribution 4.0 International License. Read Full License

Version of Record: A version of this preprint was published at BMC Cancer on April 9th, 2021. See the published version at https://doi.org/10.1186/s12885-021-08126-7. 


\section{Abstract}

Background: Definitive diagnosis of peripheral pulmonary lesions (PPLs) depends upon histologic proof obtained via pleural biopsy. Ultrasound-guided sampling is now standard practice. How to determine a suitable needle size and sampling times to improve efficacy and safety remained challenging. This study aimed to compare the efficacy for histology diagnosis and the resulting complications between 16-gauge and 18-gauge core biopsy needle in ultrasound-guided percutaneous transthoracic biopsy for PPLs.

Materials and Methods:1169 patients (767 men, 402 women; mean age, 59.4 \pm 13.2 years) who received biopsy for PPLs between September2011 and February 2019 were included. Propensity score matching (PSM) analysis was performed to adjust the baseline differences, and the rate of successful specimen assessment and complications were compared between 16-gauge (249 patients) and 18-gauge (920 patients) group. The number of pleural surfaces crossed (NOPSC) was defined as the times visceral pleural surface was transgressed. Stratified analysis was made based on NOPSC.

Results: The overall success rate was $92.0 \%$ (1076/1169). The overall complication rate was $9.6 \%$, including pneumothorax, hemorrhage and vasovagal reaction, which occurred in $2.5 \%$ (29/1169), $6.6 \%$ (77/1169), and $0.5 \%(6 / 1169)$ of patients, respectively. When NOPSC was one, the success rate and complication rate in 16-gauge group were comparable to that of 18-gauge group (both $P>0.05$ ). When NOPSC was two, the success rate in 16-gauge group was significantly higher than that of 18-gauge group $(P=0.017)$, while the complication rate was comparable ( $P>0.05)$. When NOPSC was more than two, the rate of vasovagal reaction was significantly higher in 16-gauge group than that of 18-gauge group $(P=0.012)$.

Conclusion: Higher success rate could be achieved using 16-gauge than 18-gauge core biopsy needle in ultrasound-guided percutaneous transthoracic needle biopsy for PPLs when NOPSC was two. However, the rate of vasovagal reaction would be increased by using 16-gauge needle when NOPSC was more than two.

\section{Background}

The widespread application of computed tomography (CT) in the chest has resulted in a rapid growth in the detection of pulmonary nodules, which may be frequently classified as indeterminate according to morphology [1]. Recently, since people have attached importance to screening for lung cancer, asymptomatic pulmonary nodules were more popularly noticed, and some of them were peripherally located. Peripheral pulmonary lesions (PPLs) remain a challenge to diagnosis [2-4]. Surgical biopsy, bronchoscopy, and percutaneous biopsy were frequently-used diagnosis modalities for PPLs [5]. Surgical biopsy is an authentic way to get a satisfying amount of tissue. In spite of that, it is traumatic and operated under general anesthesia [6, 7]. Bronchoscopy is relatively safe, but the diagnostic yield for PPLs was inefficient, especially when the lesions are located adjacent to the visceral pleura, with a distance under $1 \mathrm{~cm}$ [8-10]. 
Percutaneous biopsy under the guidance of ultrasound (US) or CT is more effective and less invasive than surgical biopsy $[11,12]$. CT-guided percutaneous needle biopsy for PPLs has been commonly performed in clinical practice. However, in addition to radiation exposure, it could not access real-time monitoring [13]. Besides, previous studies reported that the rate of post-procedure complications was high, including pneumothorax, hemorrhage and vasovagal reaction, which lengthened the period of hospitalization [14]. On the other hand, US-guided percutaneous needle biopsy for PPLs was more advantageous than CT-guided procedure, such as radiation-free, convenient and cost-effective, real-time and dynamic [15-17]. Therefore, US-guided biopsy for PPLs that were adjacent to visceral pleura was a potentially feasible and reliable technique [18].

Some previous studies that focused on US-guided biopsy for PPLs have verified the efficacy and safety of this technique $[16,18,19]$. However, the procedures were almost performed with 18-gauge or 20-gauge core biopsy needle. Except for patient-, lesion-, and other procedure-related factors, biopsy needle gauge may be a potential influence factor of efficacy and safety that requires further scrutiny for several reasons. First, biopsy specimens must provide enough tissue to guarantee both histologic diagnosis and immunohistochemistry analysis, and a larger needle could obtain more specimen. Second, a larger needle would be more likely to result in complication theoretically. Third, the choice of needle size is within operator control and can be easily changed without much interference to the procedural. 19-gauge and 20-gauge needle with a cutting edge to extract tissue for analysis were more commonly used in core needle biopsy [20]. With regard to needle size, larger needles may lead to increased tissue yield-in a recent ex vivo study, 16-gauge and 18-gauge needles may need fewer passes to achieve the threshold for pathologic analysis compared with smaller needles [21]. However, this assumption has not been authenticated in research with large sample so far. Information with regard to the role of size of needle in the clinical procedure is relatively limited, and how to choose a suitable core needle to balance the efficacy and safety of the procedure still remain challenging. Therefore, this study was conducted to compare the efficacy for histology diagnosis and the resulting complications between 16-gauge and 18gauge core biopsy needle in US-guided percutaneous biopsy for PPLs. To avoid possible biases and confounding factors, propensity score-matching (PSM) analysis was applied in this study [22].

\section{Materials And Methods}

\section{Patients selection}

From September 2011 to February 2019, all patients who received US-guided percutaneous transthoracic biopsy for PPLs with 16-gauge or 18-gauge core biopsy needle in our institution were included in the study. Exclusion criteria for biopsy were as follows: patients with pleural effusion, biopsy intolerance resulted from severe cough or cardiopulmonary dysfunction, the PPLs were not clearly displayed on ultrasound. Patients that carried abnormal platelet number or prolonged blood clotting time were excluded. In patients with multiple PPLs, the one that was most clearly seen and showed safe puncture path on ultrasound was selected as the main tumor for biopsy. 


\section{Data Collection}

This retrospective study was approved by the institutional review board of our hospital and informed consent was obtained from all human subjects. All procedures were performed in accordance with institutional and national standards on human experimentation, as confirmed with the Declaration of Helsinki of 1964 and its later amendments. The collected data were as follows: demographic characteristics (age, gender), patient position during procedure, the presence of emphysema, location and diameter of the lesion, and proportion of necrosis in the lesion. The pre-procedural contrast-enhanced computed tomography (CECT) was evaluated by two radiologists with 5 years of experience independently, and discrepancy was settled by discussion until consensus was reached. Diameter of lesion was determined by the long-axis measurement in the axial plane on CT. Proportion of necrosis in the lesions, which manifested as non-enhanced areas on CECT, was recorded into two groups $(<50 \%$ and $\geq 50 \%$ ).

\section{US-guided percutaneous needle biopsy}

All patients had received CECT examinations prior to the procedure to confirm location of the lesion and to obtain a feasible sonographic window before biopsy. Lesion location and characteristics were assessed on CT images which were performed before biopsy, and suitable patient positioning was determined. Patient position (supine, prone or lateral) was decided based on the location of the PPL and planning puncture pathway. Biopsies were performed under US guidance by an interventional physician with 5 years of experience. The US equipment used in this study was a MyLab Twice machine (Esoate, Genoa, Italy) equipped with a convex array probe CA541 (frequency range from 1 to $8 \mathrm{MHz}$ ). The biopsy was performed with a core needle (Figure 1). The 18-gauge core needle (Bard, Arizona, USA) was used for the procedures from September 2011 to July 2017, and the 16-gauge core needle (Bard, Arizona, USA) was then used till February 2019.

Biopsy site was disinfected and local anesthesia was given. Real-time color Doppler imaging was used to avoid vessels. Freehand technique was employed in all cases. The needle was introduced and gently advanced toward the lesion of interest. When the needle was advanced in the lesion, the biopsy was performed and the needle was then removed. The protruding needle $(22 \mathrm{~mm})$ with biopsy notch $(16 \mathrm{~mm}$ length) was the same in all cases. Biopsies were performed during patient breath-holding. Enhancing solid part of the lesions on CECT was assumed to be the targeted area for biopsy. The non-enhancing area were avoided as far as possible. Samples were assessed visually for adequacy. If the specimens were too fragmented to be processed for histological examination, biopsy was repeated until adequate specimens were achieved. Biopsies were performed 1 to 4 times as tolerated by the patient. The number of pleural surfaces crossed (NOPSC) was defined as the times visceral pleural surface was transgressed. The sample was conserved in a formalin container and delivered to histopathology analysis. 


\section{Complication evaluations}

After the procedure, the patients were introduced to kept in bed without movement getting out of bed for at least 6 hours post-procedurally. The patient's vital signs and symptoms, hemoglobin and imaging were closely observed for at least 24 hours. Chest radiograph and ultrasound were performed 1 hour after the procedure to detect complications such as pneumothorax and hemothorax and if needed, further followup radiographs were performed $[23,24]$. Vasovagal reaction frequently happens during the procedure, manifesting as either lightheadedness, hypotension, nausea, and/or transient bradycardia [25, 26].

The criterion of a large pneumothorax is the existence of a visible rim of $>2 \mathrm{~cm}$ between the lung margin and inner chest wall, which could be measured on a chest radiograph at the level of the hilum [27]. The severity of hemorrhage was divided into minor and major hemorrhage based on free fluid area detected with ultrasonography. Minor hemorrhage was defined as hemorrhage $<2 \mathrm{~cm}$ in width or only hemoptysis that did not cause obvious shortness of breath and blood oxygen saturation reduction, while major hemorrhage was defined as hemorrhage $>2 \mathrm{~cm}$. Chest tube placement was based upon the seriousness of symptoms and/or the degree of lung compression. The tube was removed when the symptom, vital sign and chest radiograph showed complication was relieved. Chest radiograph was performed prior to discharge.

\section{Pathological evaluations}

Biopsy samples were routinely embedded in paraffin. Thin sections were cut and stained with hematoxylin and eosin. Immunohistochemical analysis was performed if needed. Microscopic sections were reviewed by two pathologists with 10 years' experience of pulmonary pathology, and final diagnosis was determined when consensus was reached.

Histological findings of biopsy samples were divided into 3 groups: malignant group, benign group, and non-diagnostic group. Malignant group included any malignant diseases. The lung lesion was categorized into benign group according to the identification of definite benign features, such as tuberculoma, organizing pneumonia, chronic inflammation, granulomatous inflammation, abscess, pulmonary mycosis or other benign tumors. Results were considered non-diagnostic when the biopsy specimen was deemed insufficient for diagnosis or showed few atypical cells. The findings of malignant group and benign group were considered successful biopsies.

\section{Statistical analysis}

The statistical analysis was performed using the SPSS 22.0 software package (SPSS Inc, Chicago, IL, USA). Chi-square test or Fisher's exact test was applied to compare the difference of categorical variable. Quantitative data were expressed as mean \pm standard deviation. The difference of quantitative variable was detected by Independent Sample T test or Mann-Whitney $\mathrm{U}$ test. $P<0.05$ was considered to indicate a 
statistically significant difference. To reduce the bias from baseline confounding variables, propensityscore matching (PSM) analysis was carried out. Due to the variety in the baseline characteristics between the two groups (Table 3), PSM was performed to recognize a cohort of participants with similar baseline characteristics.

The propensity score is a conditional probability of having a particular exposure (16-gauge vs 18-gauge) given a set of measured covariates at baseline. The propensity score was evaluated with a nonparsimonious multivariable logistic-regression model. 16-gauge group was served as the dependent variable, and statistically significant baseline characteristics in Table 3 were served as covariates. PSM was performed with a 1:1 matching protocol without replacement (greedy-matching algorithm. The caliper width was equal to 0.2 of the standard deviation of the logit of the propensity score. After PSM, the baseline characteristics were compared between both groups to re-evaluate the comparability.

\section{Results}

\section{Patients and lesions profile}

A total of 1169 patients were enrolled in this study. The baseline characteristics and lesions profile were summarized in Table 1. There were 767 (65.6\%) men and 402 (34.4\%) women, with a mean age of $59.4 \pm 13.2$ years (range 18 85 years). Of these, $249(21.3 \%)$ and 920 (78.7\%) patients received biopsies with 16-gauge and 18-gauge needle, respectively. The average diameter of the lesions was $5.1 \pm 2.6 \mathrm{~cm}$ (ranging from $0.7 \mathrm{~cm}$ to $11.6 \mathrm{~cm}$ ). In 1169 patients, there was no significant difference between the16gauge $(\mathrm{n}=249)$ and 18 -gauge group $(\mathrm{n}=920)$ in gender $(P=0.268)$, patient position $(P=0.719)$, location of PPL $(P=0.590)$ and proportion of necrosis in the lesion $(P=0.184)$. However, mean age $(P=0.028)$ and mean diameter of the lesions ( $P=0.024)$ were significantly higher in the 16-gauge group. Besides, there was significant difference in number of pleural surfaces crossed $(P<0.001)$ and emphysema by CT $(P=0.044)$.

In 93 patients whose NOPSC was one, there was no significant difference between the 16-gauge $(\mathrm{n}=37)$ and 18-gauge group $(\mathrm{n}=56)$ in age $(P=0.975)$, gender $(P=0.400)$, patient position $(P=0.780)$, emphysema by CT $(P=0.254)$, location of PPL $(P=0.731)$, diameter of PPL $(P=0.447)$, and proportion of necrosis in the lesion $(P=1.000)$ (Table 2).

In 749 patients whose NOPSC was two, there was significant difference in age $(P=0.016)$ and diameter of PPL $(P=0.025)$ between the 16-gauge $(n=147)$ and 18-gauge group $(n=647)$. There was no significant difference in gender $(P=0.424)$, patient position ( $P=0.846)$, emphysema by CT ( $P=0.308)$, location of PPL $(P=0.831)$, and proportion of necrosis in the lesion $(P=0.425)$ between two groups. PSM was subsequently performed to balance the difference between the two groups. After matching, a total of 145 patient pairs (1:1) were extracted, with no significant differences in baseline characteristics between the patients in these two groups (all $P>0.05$ ) (Table 3 ). 
In 282 patients whose NOPSC was more than two, there was no significant difference between the 16gauge $(\mathrm{n}=65)$ and 18-gauge group $(\mathrm{n}=217)$ in age $(P=0.719)$, gender $(P=0.211)$, patient position $(P=0.626)$, emphysema by CT $(P=0.541)$, location of PPL $(P=0.071)$, diameter of PPL $(P=0.475)$, and proportion of necrosis in the lesion $(P=0.753)$ (Table 4).

\section{Efficacy of US-guided core needle biopsy}

The overall success rate of biopsy was $92.0 \%$ (1076/1169). Among all biopsies, 678 PPLs were categorized as malignant: 581 non-small cell lung cancers, 43 metastatic lung cancers, 31 small cell lung cancers, 11 malignant lymphomas, 9 malignant mesenchymal tumors, and 3 malignant solitary fibromas. 398 PPLs were categorized as benign: 197 chronic nonspecific inflammation, 104 tuberculoma, 37 organizing pneumonias, 18 chronic granulomatous inflammation, 13 abscesses,

17 pulmonary mycosis, 6 benign solitary fibrous tumors, 4 inflammatory pseudotumor, and 2 benign spindle cell tumors. The remaining 93 biopsy samples were categorized as non-diagnostic. The success rate of biopsy of the 16-gauge group $(96.4 \%, 240 / 249)$ was significantly higher than that of the 18-gauge group $(90.9 \%, 836 / 920)$.

The data were divided into 3 groups according to NOPSC (NOPSC $=1$; NOPSC $=2$; NOPSC $>2$ ). When NOPSC was 2 , the success rate of the 16-gauge group was significantly higher than that of the 18-gauge group in both overall cohort and PSM cohort (both $P=0.017$ ). When NOPSC was either 1 or more than 2 , the success rate of the 16-gauge group was comparable to that of the 18-gauge group ( $P=1.000$ and $P=0.254$, respectively).

\section{Complications of US-guided core needle biopsy}

The overall post-procedure complication rate was $9.6 \%$ (112/1169). None of these incidents resulted in permanent severe sequelae or death. The rate of complication in the 16-gauge group $(9.6 \%, 24 / 249)$ were comparable to that in the 18-gauge group $(9.6 \%, 88 / 920)(P=0.972)$. Totally, pneumothorax occurred in 29 out of 1169 patients (2.5\%), including 22 cases of minor pneumothorax and 7 cases of major pneumothorax. When minor pneumothorax was detected, air was escaped from the pleural during needle removal. Patients were then monitored in a puncture-site-down position immediately and treated with nasal oxygen for at least 3 hours. Major pneumothorax was observed in 7 patients $(0.6 \%)$ who required post-procedural treatment via pigtail catheter insertion. There was no significant difference in the rate of pneumothorax between 16 -gauge and 18 -gauge group (1.6\% vs $2.7 \%, P=0.317$ ).

The overall rate of hemorrhage was $6.6 \%$ (77/1169). No major hemorrhage occurred. All of the hemorrhages were reported as minor that were on observation to be stable without deterioration. Minor hemorrhage occurred with or without hemoptysis. Pleural effusion and hemoptysis were recorded in 16 and 61 patients, respectively. In this study, the treatment of hemorrhage included placement in a puncture-site-down position with the bleeding lung placed downward to make the non-operated lung free 
air accessible and Tranexamic acid 500-1,000 mg intra-venously (non-bolus). There was no significant difference in the rate of hemorrhage between 16 -gauge and 18 -gauge group ( $6.0 \%$ vs $6.8 \%, P=0.687)$.

The overall rate of vasovagal reaction was $0.5 \%$ (6/1169). The reactions were managed by stopping the procedure immediately, placing the patient in a recumbent position and elevating the lower extremities. The vital sign and consciousness were monitored closely, and the recovery time was quick. The rate of vasovagal reaction was significantly higher in the 16-gauge group than that of 18 -gauge group ( $2.0 \%$ vs $0.1 \%, P=0.002)$.

When NOPSC was 1, the rates of pneumothorax, hemorrhage and vasovagal reaction in the 16-gauge group were comparable to that of the 18-gauge group (all P>0.05). When NOPSC was 2 , the rates of pneumothorax, hemorrhage and vasovagal reaction in the 16-gauge group were comparable to that of the 18-gauge group in both overall cohort and PSM cohort (all $P>0.05$ ). When NOPSC was more than 2, there was no significant difference in the rates of pneumothorax and hemorrhage between 16-gauge and 18gauge group (both $P>0.05$ ), while the rate of vasovagal reaction was significantly higher in the 16-gauge group than that of 18 -gauge group $(6.2 \%$ vs $0.4 \%, P=0.012)$.

\section{Discussion}

Determining needle size to be used in biopsy for PPLs is an important consideration for radiologists who perform thoracic interventions. Selecting the safest and most effective needle should be a priority. In previous studies regarding US-guided percutaneous biopsy for PPLs, the procedures were mainly performed with 18-gauge or smaller size needle, but the insufficient tissue yield still remained a challenge. Besides, how to determine needle size and NOPSC had not been explored thoroughly. The current study confirmed the high efficacy and safety of US-guided percutaneous transthoracic biopsy for PPLs and revealed that US-guided biopsy had a higher success rate without increased complications by using 16gauge needle when NOPSC was two. Pneumothorax and hemorrhage rate did not raise with 16-gauge needle compared with 18-gauge needle. However, the rate of post-procedural vasovagal reaction would be increased by using 16-gauge needle when NOPSC was more than two.

US-guided percutaneous biopsy has been achieving gradual acceptance in clinical practice. Compared with CT-guided procedures, US guidance had advantages such as no radiation, real-time monitoring, convenient and low-cost. Importantly, it had success rates of $81.8 \% \sim 93.4 \%$ in previous studies[28-30]. In our study, the success rate of diagnosis for biopsy was $92.0 \%$. According to the present study, the successful diagnostic rate of 16-gauge needle presented significant advantages over 18-gauge needle when NOPSC was two. The trend toward improved pathologic sample success with 16-gauge needle mainly resulted from the ability to directly visualize sampling of PPLs in US real time guidance and the use of a larger gauge needle for core biopsy. It is not difficult to understand that the volume of samples is larger if a 16-gauge needle is applied in clinical practice. As is known to all, repeatability and a larger number of samples may increase the success rate. However, the most suitable NOPSC to reach adequacy threshold for successful diagnosis with 16-gauge needle still remains unclear. In this study, a 16-gauge 
core biopsy needle was employed in about $21 \%$ of cases, yielding a larger tissue sample and improving the success rate of pathological evaluation in cases with two pleural surfaces crossed. Although age and diameter of PPLs were significantly unbalanced between 16-gauge and 18-gauge group in stratified analysis of two pleural surfaces crossed, PSM was utilized to select cases, reduce the bias from confounding variables and balance baseline characteristics between two groups. In summary, 16-gauge needle with two punctured samplings should be recommended for biopsy in consideration for its higher efficacy compared with 18-gauge needle.

US-guided biopsies were safe with an overall complication rate of $9.6 \%$ in this study. No death or severe sequela resulted from the procedures. These findings were acceptable compared with those reported in previous studies, which occurred in $2.1 \%$ 12.8\% of patients[28, 29, 31]. However, Guo et al reported an overall complication rate of $12.8 \%$ in 637 PPLs, in the situation of 16 -gauge needle applied in $24 \%$ of lesions and 18-gauge needle applied in $76 \%$ of lesions [31]. In the study of Guo et al, more needle passes might contribute to the slightly higher rate compared with the current study. The median NOPSC reached more than three in the study of Guo et al while it was two in our study. Furthermore, Guo et al reported hemorrhage and pneumothorax occurred in $8.0 \%$ and $1.7 \%$ of cases, respectively [31], which were similar to our study. On the other hand, only $0.6 \%$ of patients suffered from post-procedure pneumothorax that required chest catheter insertion and recovered within $3 \sim 5$ days. In this study, hemoptysis was selflimiting, which could be relieved with patient reassurance and positioned laterally with the biopsy side down. Hemorrhage in the absence of hemoptysis was usually minor and often asymptomatic, but the patient would present becoming confused from hypoxia or shocked if larger bleeding occurred. In the case of severe bleeding, patients may receive bronchoscopy with tamponade, a balloon catheter, coagulopathies and coiling. Vasovagal reaction is a relatively rare complication caused by reflex vagal hyperfunction induced by pleural stimulation, and it often does not required atropine. Pereyra et al reported vasovagal reactions occurred in 27 of 678 blind closed biopsies for pleural biopsy, for an incidence of $4.1 \%$ [26]. In our study, the rate was only $0.6 \%$, mainly because of rapid puncture across the pleura when performing biopsy to reduce the period of provoking the pleura.

In addition to the efficacy benefits, overall complication rate was analogous between 16-gaugeand 18gauge needle. Many of the factors that have been previously proved to predispose to complication are patient or lesion related and could not be changed [19, 32]. Needle size, however, is well within operator control. To the best of our knowledge, previous studies have rarely compared the safety between 16gauge and 18-gauge core biopsy needle. It was thought that complications increased with larger needle size, which tended to injure a larger part of lung parenchyma and lead to expanding in the amount of air leakage and bleeding. However, the results demonstrated that the rates of pneumothorax and haemorrhage were not higher in 16-gauge than in 18-gauge group. Only when NOPSC was more than two, the incidence of vasovagal reaction was higher in 16-gauge group. Besides, in 16-gauge group, the success rates were comparable between NOPSC $=2$ and NOPSC $>2$ groups $(P=0.723)$. Compared with NOPSC $>2$, although the complication rate was not significantly higher when NOPSC $=2(16.9 \%$ vs $8.2 \%$, $P=0.059)$, there was an observed uptrend. Therefore, to reduce complication occurrence and increase success rate, 16-gauge core needle with two punctured samplings was recommend as the routine use. 
There were several limitations to our study. First, selection bias was inevitable as a retrospective study and patients were not randomized to 16-gauge or 18-gauge group, which may result in selection bias, although the impact was attempted to minimize using PSM analysis. Second, the data originated from a single center. The results in this study might not represent the experiences of other institutions. Besides, other potential risk factors that did not include in the study could not be evaluated for confounding effects, and comparison with CT-guided biopsy was not performed. Therefore, further validation of results of this study is still warranted.

\section{Conclusions}

US-guided percutaneous transthoracic biopsy for PPLs is an effective and safe procedure. Higher success rate could be achieved by using 16-gauge compared with 18-gauge core biopsy needle when NOPSC was two. However, the rate of post-procedural vasovagal reaction would be increased by using 16-gauge needle when NOPSC was more than two.

\section{Abbreviations}

PPLs: Peripheral pulmonary lesions; PSM: Propensity score matching; NOPSC: Number of pleural surfaces crossed; CT: Computed tomography; US: Ultrasound; CECT: Contrast-enhanced computed tomography

\section{Declarations}

\section{Authors' contributions}

Qiu YD, Peng WW, Lan NH, Cui WZ, Huang T and Ou YH participated in literature search, data acquisition, data analysis, or data interpretation. Huang WJ, Ye JY and Li YJ conceived and designed the study, and critically revised the manuscript, performed the research, wrote the first draft, collected and analyzed the data. Huang WJ and Ye JY participated in paper writing and revised the manuscript. All authors have read and approved the manuscript.

\section{Funding}

This work was supported by the Foshan Science and technology innovation project [Grant No. 202001004115].

\section{Availability of data and materials}

All the data and materials supporting the conclusions were included in the main paper. The datasets used in the current study could be available from the corresponding author on request. 


\section{Ethics approval and consent to participate}

This study was approved by the Institutional Review Board of First people's Hospital of Foshan, and informed consent was obtained from all human subjects.

\section{Consent for publication}

Not applicable.

\section{Competing interests}

All authors declare that they have no competing interests.

\section{References}

1. Cheng TY, Cramb SM, Baade PD, Youlden DR, Nwogu C, Reid ME: The International Epidemiology of Lung Cancer: Latest Trends, Disparities, and Tumor Characteristics. J Thorac Oncol 2016; 11:16531671.

2. Rivera MP, Mehta AC, Wahidi MM: Establishing the diagnosis of lung cancer: Diagnosis and management of lung cancer, 3rd ed: American College of Chest Physicians evidence-based clinical practice guidelines. Chest 2013; 143:e142S-e165S.

3. Loverdos K, Fotiadis A, Kontogianni C, Iliopoulou M, Gaga M: Lung nodules: A comprehensive review on current approach and management. Ann Thorac Med 2019; 14:226-238.

4. Khan T, Usman Y, Abdo T, Chaudry F, Keddissi JI, Youness HA: Diagnosis and management of peripheral lung nodule. Ann Transl Med 2019; 7:348.

5. Nishi SPE, Zhou J, Okereke I, Kuo Y-F, Goodwin J: Use of Imaging and Diagnostic Procedures After Low-Dose CT Screening for Lung Cancer. Chest 2020; 157:427-434.

6. Riley DJ, Costanzo EJ: Surgical biopsy: its appropriateness in diagnosing interstitial lung disease. Curr Opin Pulm Med 2006; 12:331-336.

7. Parambil JG, Savci CD, Tazelaar HD, Ryu JH: Causes and presenting features of pulmonary infarctions in 43 cases identified by surgical lung biopsy. Chest 2005; 127:1178-1183.

8. Chavez C, Sasada S, Izumo T, Watanabe J, Katsurada M, Matsumoto Y et al: Endobronchial ultrasound with a guide sheath for small malignant pulmonary nodules: a retrospective comparison between central and peripheral locations. J Thorac Dis 2015; 7:596-602.

9. Eberhardt R, Anantham D, Ernst A, Feller-Kopman D, Herth F: Multimodality bronchoscopic diagnosis of peripheral lung lesions: a randomized controlled trial. Am J Respir Crit Care Med 2007; 176:36-41.

10. Silvestri GA, Bevill BT, Huang J, Brooks M, Choi Y, Kennedy G et al: An Evaluation of Diagnostic Yield From Bronchoscopy: The Impact of Clinical/Radiographic Factors, Procedure Type, and Degree of 
Suspicion for Cancer. Chest 2020.

11. Capalbo E, Peli M, Lovisatti M, Cosentino M, Mariani P, Berti E et al: Trans-thoracic biopsy of lung lesions: FNAB or CNB? Our experience and review of the literature. Radiol Med 2014; 119:572-594.

12. Fu Y, Zhang YY, Cui LG, Tan S, Sun Y: Ultrasound-Guided Biopsy of Pleural-Based Pulmonary Lesions by Injection of Contrast-Enhancing Drugs. Front Pharmacol 2019; 10:960.

13. Fu YF, Li GC, Xu QS, Shi YB, Wang C, Wang T: Computed tomography-guided lung biopsy: a randomized controlled trial of low-dose versus standard-dose protocol. European radiology 2020; 30:1584-1592.

14. Huo YR, Chan MV, Habib AR, Lui I, Ridley L: Pneumothorax rates in CT-Guided lung biopsies: a comprehensive systematic review and meta-analysis of risk factors. Br J Radiol 2020:20190866.

15. Wang $Y, X u Z$, Huang $H$, Zhou X, Xian M: Application of quantitative contrast-enhanced ultrasound for evaluation and guiding biopsy of peripheral pulmonary lesions: a preliminary study. Clin Radiol 2020; 75:79.e19-79.e24.

16. Huang W, Chen L, Xu N, Wang L, Liu F, He S et al: Diagnostic value and safety of color doppler ultrasound-guided transthoracic core needle biopsy of thoracic disease. Biosci Rep 2019; 39.

17. Khan RA, Kumar V, Taimur M, Khan MA, Arshad MM, Amjad MA: Diagnostic Yield of Ultrasoundguided Trucut Biopsy in Diagnosis of Peripheral Lung Malignancies. Cureus 2019; 11:e4802.

18. Tekin AF, Turgut B, Öncü F: Should We Perform Transthoracic Trucut Lung Biopsies of Pleural-Based Lung Masses Under Ultrasound Guidance or Computed Tomography Guidance? Ultrasound quarterly 2020; 36:49-53.

19. Lee MH, Lubner MG, Hinshaw JL, Pickhardt PJ: Ultrasound Guidance Versus CT Guidance for Peripheral Lung Biopsy: Performance According to Lesion Size and Pleural Contact. AJR Am J Roentgenol 2018; 210:W110-w117.

20. Tam AL, Lim HJ, Wistuba, II, Tamrazi A, Kuo MD, Ziv E et al: Image-Guided Biopsy in the Era of Personalized Cancer Care: Proceedings from the Society of Interventional Radiology Research Consensus Panel. J Vasc Interv Radiol 2016; 27:8-19.

21. Hoang NS, Ge BH, Pan LY, Ozawa MG, Kong CS, Louie JD et al: Determining the Optimal Number of Core Needle Biopsy Passes for Molecular Diagnostics. Cardiovasc Intervent Radiol 2018; 41:489-495.

22. Kane LT, Fang T, Galetta MS, Goyal DKC, Nicholson KJ, Kepler CK et al: Propensity Score Matching: A Statistical Method. Clin Spine Surg 2020; 33:120-122.

23. Manhire A, Charig M, Clelland C, Gleeson F, Miller R, Moss H et al: Guidelines for radiologically guided lung biopsy. Thorax 2003; 58:920-936.

24. Perlmutt LM, Braun SD, Newman GE, Oke EJ, Dunnick NR: Timing of chest film follow-up after transthoracic needle aspiration. AJR Am J Roentgenol 1986; 146:1049-1050.

25. Jones PW, Moyers JP, Rogers JT, Rodriguez RM, Lee YC, Light RW: Ultrasound-guided thoracentesis: is it a safer method? Chest 2003; 123:418-423. 
26. Pereyra MF, San-José E, Ferreiro L, Golpe A, Antúnez J, González-Barcala F-J et al: Role of blind closed pleural biopsy in the managment of pleural exudates. Can Respir J 2013; 20:362-366.

27. Christiansen IS, Clementsen PF, Bodtger U, Naur TMH, Pietersen PI, Laursen CB: Transthoracic ultrasound-guided biopsy in the hands of chest physicians - a stepwise approach. Eur Clin Respir J 2019; 6:1579632.

28. Yamamoto N, Watanabe T, Yamada K, Nakai T, Suzumura T, Sakagami K et al: Efficacy and safety of ultrasound (US) guided percutaneous needle biopsy for peripheral lung or pleural lesion: comparison with computed tomography (CT) guided needle biopsy. J Thorac Dis 2019; 11:936-943.

29. Jeon KN, Bae K, Park MJ, Choi HC, Shin HS, Shin S et al: US-guided transthoracic biopsy of peripheral lung lesions: pleural contact length influences diagnostic yield. Acta Radiol 2014; 55:295-301.

30. Lesser TG, Petersen I, Polzing F, Wolfram F: One-Lung Flooding Enables Ultrasound-Guided Transthoracic Needle Biopsy of Pulmonary Nodules with High Sensitivity. Ultrasound Med Biol 2018; 44:1556-1562.

31. Guo YQ, Liao XH, Li ZX, Chen YY, Wang SD, Wang JH et al: Ultrasound-Guided Percutaneous Needle Biopsy for Peripheral Pulmonary Lesions: Diagnostic Accuracy and Influencing Factors. Ultrasound Med Biol 2018; 44:1003-1011.

32. Portela de Oliveira E, Souza CA, Inacio JR, Abdelzarek M, Dennie C, Gupta A et al: Imaging-guided Percutaneous Biopsy of Nodules $</=1 \mathrm{~cm}$ : Study of Diagnostic Performance and Risk Factors Associated With Biopsy Failure. J Thorac Imaging 2020; 35:123-128.

\section{Tables}

Table 1 Characteristics and lesion profile of patients undergoing ultrasound-guided lung biopsy using 16gauge and 18-gauge biopsy needle. 


\begin{tabular}{|c|c|c|c|}
\hline Characteristics & 16-gauge $(n=249)$ & 18-gauge $(n=920)$ & $P$ value \\
\hline Age (years) & $57.8 \pm 14.5$ & $60.0 \pm 12.8$ & 0.028 \\
\hline \multicolumn{4}{|l|}{ Gender } \\
\hline Male/female & $156 / 93$ & $611 / 309$ & 0.268 \\
\hline \multicolumn{4}{|l|}{ Patient position (\%) } \\
\hline Supine & $93(37.3 \%)$ & $329(35.8 \%)$ & 0.719 \\
\hline Prone & $112(45.0 \%)$ & $440(47.8 \%)$ & \\
\hline Lateral & $44(17.7 \%)$ & $151(16.4 \%)$ & \\
\hline Emphysema by CT & $19(7.6 \%)$ & $112(12.2 \%)$ & 0.044 \\
\hline \multicolumn{4}{|c|}{ Location of pulmonary lesion (\%) } \\
\hline Left upper lobe & $42(16.9 \%)$ & $193(21.0 \%)$ & 0.590 \\
\hline Left lower lobe & $64(25.7 \%)$ & $207(22.5 \%)$ & \\
\hline Right upper lobe & $64(25.7 \%)$ & $245(26.6 \%)$ & \\
\hline Right middle lobe & $21(8.4 \%)$ & $75(8.2 \%)$ & \\
\hline Right lower lobe & $58(23.3 \%)$ & $200(21.7 \%)$ & \\
\hline Diameter of lesion (cm) & $4.8 \pm 2.5$ & $5.2 \pm 2.6$ & 0.024 \\
\hline$<5$ & 119 (47.8\%) & $439(47.7 \%)$ & 0.984 \\
\hline$\geq 5$ & $130(52.2 \%)$ & $481(52.3 \%)$ & \\
\hline \multicolumn{4}{|c|}{ Proportion of necrosis in the lesion } \\
\hline$<50 \%$ & $215(86.3 \%)$ & $762(82.8 \%)$ & \multirow[t]{2}{*}{0.184} \\
\hline$\geq 50 \%$ & $34(13.7 \%)$ & $158(17.2 \%)$ & \\
\hline \multicolumn{4}{|c|}{ Number of pleural surfaces crossed } \\
\hline 1 & $37(14.9 \%)$ & $56(6.1 \%)$ & \multirow[t]{3}{*}{$<0.001$} \\
\hline 2 & 147 (59.0\%) & 647 (70.3\%) & \\
\hline$>2$ & 65 (26.1\%) & $217(23.6 \%)$ & \\
\hline Success rate (\%) & 240 (96.4\%) & $836(90.9 \%)$ & 0.004 \\
\hline Malignant & 135 (54.2\%) & $543(59.1 \%)$ & \multirow[t]{3}{*}{0.001} \\
\hline Benign & 105 (42.2\%) & $293(31.8 \%)$ & \\
\hline Non-diagnostic & $9(3.6 \%)$ & $84(9.1 \%)$ & \\
\hline
\end{tabular}




\begin{tabular}{|llll|} 
Complication rate (\%) & $24(9.6 \%)$ & $88(9.6 \%)$ & 0.972 \\
\hline Pneumothorax & $4(1.6 \%)$ & $25(2.7 \%)$ & 0.317 \\
\hline Hemorrhage & $15(6.0 \%)$ & $62(6.8 \%)$ & 0.687 \\
\hline Vasovagal reaction & $5(2.0 \%)$ & $1(0.1 \%)$ & 0.002 \\
\hline
\end{tabular}

Table 2 Comparison of characteristics, efficacy and safety in patients undergoing ultrasound-guided lung biopsy between 16-gauge and 18-gauge core biopsy needle when number of pleural surface crossed was 1. 


\begin{tabular}{|c|c|c|c|}
\hline Variable & 16-gauge $(n=37)$ & 18-gauge $(n=56)$ & $P$ value \\
\hline Age (years) & $59.0 \pm 13.8$ & $59.0 \pm 13.3$ & 0.975 \\
\hline \multicolumn{4}{|l|}{ Gender } \\
\hline Male/female & $25 / 12$ & $33 / 23$ & 0.400 \\
\hline \multicolumn{4}{|l|}{ Patient position } \\
\hline Supine/prone/lateral & $16 / 13 / 8$ & $27 / 30 / 9$ & 0.780 \\
\hline Emphysema by CT & $0(0 \%)$ & $4(7.1 \%)$ & 0.254 \\
\hline \multicolumn{4}{|c|}{ Location of pulmonary lesion (\%) } \\
\hline Left upper lobe & $10(27.1 \%)$ & $16(28.6 \%)$ & \multirow[t]{5}{*}{0.731} \\
\hline Left lower lobe & $9(24.3 \%)$ & $10(17.9 \%)$ & \\
\hline Right upper lobe & $7(18.9 \%)$ & $14(25.0 \%)$ & \\
\hline Right middle lobe & $2(5.4 \%)$ & $6(10.6 \%)$ & \\
\hline Right lower lobe & $9(24.3 \%)$ & $10(17.9 \%)$ & \\
\hline Diameter of lesion (cm) & $4.3 \pm 2.2$ & $4.7 \pm 2.4$ & 0.447 \\
\hline$<5 / \geq 5$ & $21 / 16$ & $26 / 30$ & 0.330 \\
\hline \multicolumn{4}{|c|}{ Proportion of necrosis in the lesion } \\
\hline$<50 \% / \geq 50 \%$ & $35 / 2$ & $52 / 4$ & 1.000 \\
\hline Success rate (\%) & $36(97.3 \%)$ & $54(96.4 \%)$ & 1.000 \\
\hline Malignant & $23(62.2 \%)$ & $28(50.0 \%)$ & \multirow[t]{3}{*}{0.514} \\
\hline Benign & $13(35.1 \%)$ & $26(46.4 \%)$ & \\
\hline Non-diagnostic & $1(2.7 \%)$ & $2(3.6 \%)$ & \\
\hline Complication rate (\%) & $1(2.7 \%)$ & $8(14.3 \%)$ & 0.081 \\
\hline Pneumothorax & $0(0 \%)$ & $3(5.4 \%)$ & 0.406 \\
\hline Major & $0(0 \%)$ & $0(0 \%)$ & - \\
\hline Minor & $0(0 \%)$ & $3(5.4 \%)$ & 0.406 \\
\hline Hemorrhage & $1(2.7 \%)$ & $5(8.9 \%)$ & 0.397 \\
\hline Major & $0(0 \%)$ & $0(0 \%)$ & - \\
\hline Minor & $1(2.7 \%)$ & $5(8.9 \%)$ & 0.397 \\
\hline Vasovagal reaction & $0(0 \%)$ & $0(0 \%)$ & - \\
\hline
\end{tabular}


Table 3 Comparison of characteristics, efficacy and safety in patients undergoing ultrasound-guided lung biopsy between 16-gauge and 18-gauge core biopsy needle when number of pleural surface crossed was 2 . 


\begin{tabular}{|c|c|c|c|c|c|c|}
\hline \multirow[b]{2}{*}{ Variable } & \multicolumn{3}{|c|}{ Overall cohort } & \multicolumn{3}{|c|}{ Propensity-score matched cohort } \\
\hline & $\begin{array}{l}\text { 16-gauge } \\
(\mathrm{n}=147)\end{array}$ & $\begin{array}{l}\text { 18-gauge } \\
(\mathrm{n}=647)\end{array}$ & $\begin{array}{l}P \\
\text { value }\end{array}$ & $\begin{array}{l}\text { 16-gauge } \\
(\mathrm{n}=145)\end{array}$ & $\begin{array}{l}\text { 18-gauge } \\
(n=145)\end{array}$ & $\begin{array}{l}P \\
\text { value }\end{array}$ \\
\hline Age (years) & $57.8 \pm 14.2$ & $60.6 \pm 12.4$ & 0.016 & $58.3 \pm 13.6$ & $58.1 \pm 13.3$ & 0.889 \\
\hline \multicolumn{7}{|l|}{ Gender } \\
\hline Male/female & $94 / 53$ & $436 / 211$ & 0.424 & $94 / 51$ & $96 / 49$ & 0.805 \\
\hline \multicolumn{7}{|l|}{ Patient position } \\
\hline Supine/prone/lateral & $52 / 69 / 26$ & 232/313/102 & 0.846 & $51 / 69 / 25$ & $48 / 74 / 23$ & 0.840 \\
\hline Emphysema by CT & $16(10.9 \%)$ & $91(14.1 \%)$ & 0.308 & $\begin{array}{l}16 \\
(11.0 \%)\end{array}$ & $\begin{array}{l}18 \\
(12.4 \%)\end{array}$ & 0.715 \\
\hline \multicolumn{7}{|l|}{$\begin{array}{l}\text { Location of pulmonary } \\
\text { lesion (\%) }\end{array}$} \\
\hline Left upper lobe & $27(18.4 \%)$ & $128(19.8 \%)$ & 0.831 & $\begin{array}{l}27 \\
(18.7 \%)\end{array}$ & $\begin{array}{l}31 \\
(21.4 \%)\end{array}$ & 0.465 \\
\hline Left lower lobe & $35(23.8 \%)$ & $144(22.2 \%)$ & & $\begin{array}{l}34 \\
(23.4 \%)\end{array}$ & $\begin{array}{l}42 \\
(29.0 \%)\end{array}$ & \\
\hline Right upper lobe & $34(23.1 \%)$ & $174(26.9 \%)$ & & $\begin{array}{l}34 \\
(23.4 \%)\end{array}$ & $\begin{array}{l}32 \\
(22.0 \%)\end{array}$ & \\
\hline Right middle lobe & $12(8.2 \%)$ & $51(7.9 \%)$ & & $12(8.3 \%)$ & $14(9.7 \%)$ & \\
\hline Right lower lobe & $39(26.5 \%)$ & $150(23.2 \%)$ & & $\begin{array}{l}38 \\
(26.2 \%)\end{array}$ & $\begin{array}{l}26 \\
(17.9 \%)\end{array}$ & \\
\hline $\begin{array}{l}\text { Diameter of lesion } \\
(\mathrm{cm})\end{array}$ & $4.8 \pm 2.6$ & $5.3 \pm 2.6$ & 0.025 & $4.8 \pm 2.6$ & $5.0 \pm 2.4$ & 0.582 \\
\hline$<5 / \geq 5$ & $70 / 77$ & $313 / 334$ & 0.868 & $68 / 77$ & $69 / 76$ & 0.906 \\
\hline \multicolumn{7}{|l|}{$\begin{array}{l}\text { Proportion of necrosis } \\
\text { in the lesion }\end{array}$} \\
\hline$<50 \% / \geq 50 \%$ & $123 / 24$ & $523 / 124$ & 0.425 & $121 / 24$ & $113 / 32$ & 0.234 \\
\hline Success rate (\%) & $\begin{array}{l}141 \\
(95.9 \%)\end{array}$ & $580(89.6 \%)$ & 0.017 & $\begin{array}{l}139 \\
(95.9 \%)\end{array}$ & $\begin{array}{l}128 \\
(88.3 \%)\end{array}$ & 0.017 \\
\hline Malignant & 76 (51.7\%) & $393(60.7 \%)$ & $<0.001$ & $\begin{array}{l}76 \\
(52.4 \%)\end{array}$ & $\begin{array}{l}89 \\
(61.4 \%)\end{array}$ & 0.003 \\
\hline Benign & $65(44.2 \%)$ & $187(28.9 \%)$ & & $\begin{array}{l}63 \\
(43.5 \%)\end{array}$ & $\begin{array}{l}39 \\
(26.9 \%)\end{array}$ & \\
\hline Non-diagnostic & $6(4.1 \%)$ & $67(10.4 \%)$ & & $6(4.1 \%)$ & $17(9.7 \%)$ & \\
\hline Complication rate (\%) & $12(8.2 \%)$ & $56(8.7 \%)$ & 0.847 & $11(7.6 \%)$ & $10(6.9 \%)$ & 0.821 \\
\hline
\end{tabular}




\begin{tabular}{|lllllll|} 
Pneumothorax & $3(2.0 \%)$ & $14(2.2 \%)$ & 1.000 & $3(2.1 \%)$ & $4(2.8 \%)$ & 1.000 \\
\hline Major & $2(1.4 \%)$ & $2(0.3 \%)$ & 0.158 & $2(1.4 \%)$ & $1(0.7 \%)$ & 1.000 \\
\hline Minor & $1(0.7 \%)$ & $12(1.9 \%)$ & 0.514 & $1(0.7 \%)$ & $3(2.1 \%)$ & 0.615 \\
\hline Hemorrhage & $8(5.5 \%)$ & $44(6.8 \%)$ & 0.209 & $7(4.8 \%)$ & $6(4.1 \%)$ & 0.777 \\
\hline Major & $0(0 \%)$ & $0(0 \%)$ & - & $0(0 \%)$ & $0(0 \%)$ & - \\
\hline Minor & $8(5.5 \%)$ & $44(6.8 \%)$ & 0.548 & $7(4.8 \%)$ & $6(4.1 \%)$ & 0.777 \\
Vasovagal reaction & $1(0.7 \%)$ & $0(0 \%)$ & 0.185 & $1(0.7 \%)$ & $0(0 \%)$ & 1.000 \\
\hline
\end{tabular}

Table 4 Comparison of characteristics, efficacy and safety in patients undergoing ultrasound-guided lung biopsy between 16-gauge and 18-gauge core biopsy needle when number of pleural surface crossed was more than 2. 


\begin{tabular}{|c|c|c|c|}
\hline Variable & 16-gauge $(n=65)$ & 18-gauge $(n=217)$ & $P$ value \\
\hline Age (years) & $57.1 \pm 15.9$ & $57.8 \pm 13.7$ & 0.719 \\
\hline \multicolumn{4}{|l|}{ Gender } \\
\hline Male/female & $37 / 28$ & $142 / 75$ & 0.211 \\
\hline \multicolumn{4}{|l|}{ Patient position } \\
\hline Supine/prone/lateral & $25 / 30 / 10$ & $70 / 107 / 40$ & 0.626 \\
\hline Emphysema by CT & $3(4.6 \%)$ & $17(7.8 \%)$ & 0.541 \\
\hline \multicolumn{4}{|c|}{ Location of pulmonary lesion (\%) } \\
\hline Left upper lobe & $5(7.7 \%)$ & $49(22.6 \%)$ & \multirow[t]{5}{*}{0.071} \\
\hline Left lower lobe & $20(30.8 \%)$ & $53(24.4 \%)$ & \\
\hline Right upper lobe & $23(35.3 \%)$ & $57(26.3 \%)$ & \\
\hline Right middle lobe & $7(10.8 \%)$ & $18(8.3 \%)$ & \\
\hline Right lower lobe & $10(15.4 \%)$ & $40(18.4 \%)$ & \\
\hline Diameter of lesion (cm) & $5.0 \pm 2.3$ & $5.2 \pm 2.6$ & 0.475 \\
\hline$<5 / \geq 5$ & $28 / 37$ & $100 / 117$ & 0.669 \\
\hline \multicolumn{4}{|c|}{ Proportion of necrosis in the lesion } \\
\hline$<50 \% / \geq 50 \%$ & $57 / 8$ & $187 / 30$ & 0.753 \\
\hline Success rate (\%) & $63(96.9 \%)$ & $202(93.1 \%)$ & 0.254 \\
\hline Malignant & $36(55.4 \%)$ & $122(56.2 \%)$ & \multirow[t]{3}{*}{0.469} \\
\hline Benign & $27(41.5 \%)$ & $80(36.9 \%)$ & \\
\hline Non-diagnostic & $2(3.1 \%)$ & $15(6.9 \%)$ & \\
\hline Complication rate (\%) & $11(16.9 \%)$ & $22(10.1 \%)$ & 0.135 \\
\hline Pneumothorax & $1(1.5 \%)$ & $8(3.7 \%)$ & 0.690 \\
\hline Major & $1(1.5 \%)$ & $2(0.9 \%)$ & 0.546 \\
\hline Minor & $0(0 \%)$ & $6(3.8 \%)$ & 0.387 \\
\hline Hemorrhage & $6(9.2 \%)$ & $13(6.0 \%)$ & 0.398 \\
\hline Major & $0(0 \%)$ & $0(0 \%)$ & - \\
\hline Minor & $6(9.2 \%)$ & $13(6.0 \%)$ & 0.398 \\
\hline Vasovagal reaction & $4(6.2 \%)$ & $1(0.4 \%)$ & 0.012 \\
\hline
\end{tabular}




\section{Figures}

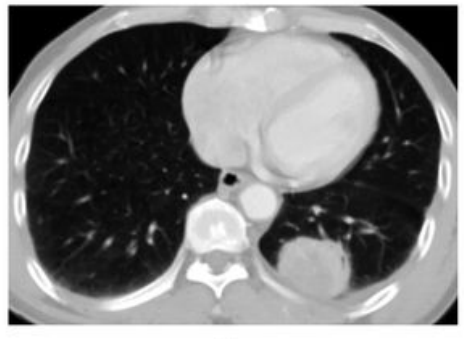

A

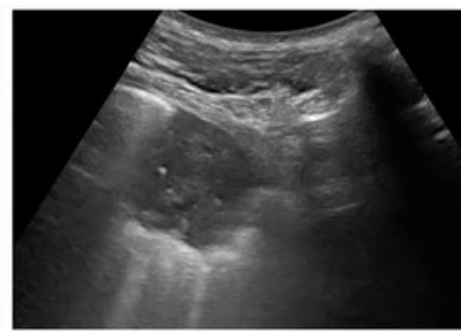

B

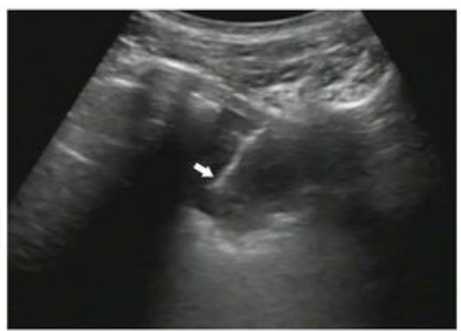

c

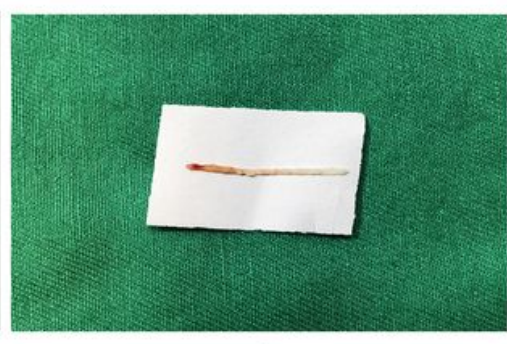

D

Figure 1

A patient aged between 55 to 60 years old with a peripheral lung lesion. Percutaneous core biopsy was requested to determine the nature of the lesion. a: Axial contrast-enhanced CT image showed a round lung lesion located in left lower lobe with pleural contact. b: Transverse gray-scale ultrasound (US) image obtained before biopsy showed that the lesion was hypoechoic relative to surrounding aerated lung. Positioning transducer in adjacent rib space showed broad pleural contact, providing sonographic window for sampling. c: Transverse gray-scale US image showed 16-gauge needle throw for core biopsy within the lesion (arrow), which was performed within single breath-hold. d: Color photograph of the biopsy sample. 